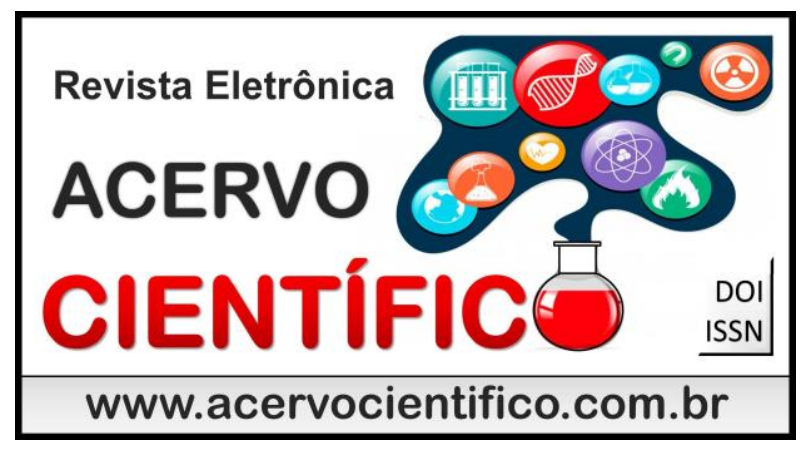

Recebido em: 12/2018

Aceito em: 12/2018

Publicado em: 01/2019

\title{
TUMOR SARCOMATOSO EXTERIORIZADO POR ORIFÍCIO CERVICAL UTERINO (SARCOMA PARIDO): RELATO DE CASO
}

\author{
Sarcomatosum Tumor exteriorized by Uterine Cervix (Born Sarcoma): Case Report. \\ Tumor Sarcomatoso Exteriorizado por el Orifício Cervical Uterino (Sarcoma Parido): \\ Relato de Caso
}
Mariana Pimentel Lopes ${ }^{1}$, Marília Monte ${ }^{1}$, Thais Sousa Rodrigues ${ }^{1,2}$, Raíssa Veloso Prado Napoleão Braz ${ }^{2 \star}$, Mykaelly Kelly de Sá Carvalho ${ }^{1}$

Resumo: Paciente do sexo feminino, 62 anos, com história de sangramento vaginal na pós-menopausa, abundante e intermitente, com duração de seis meses, associado à perda ponderal importante, dor pélvica e dificuldade miccional. Exames complementares evidenciaram lesão expansiva em istmo e colo uterino, com sinais de invasão parametrial bilateral e terço superior da vagina, em íntimo contato com bexiga e reto. A biópsia constatou tratar-se de sarcoma fusocelular de alto grau. A paciente foi encaminhada para quimioterapia e radioterapia neoadjuvante com programação para posterior tratamento cirúrgico. Este caso corrobora com o pensamento que embora raro, é importante aventar a possibilidade de sarcoma uterino, em casos de sangramento vaginal na pós menopausa. É fundamental estudo histológico e estadiamento para obter-se uma melhor qualidade terapêutica.

Palavras-chave: hemorragia uterina, neoplasia uterina, sarcoma.

\begin{abstract}
A female patient, 62 years old, with a history of postmenopausal vaginal bleeding, abundant and intermittent, lasting six months, associated with significant weight loss, pelvic pain and voiding difficulty. Complementary exams revealed an expansive lesion in the isthmus and cervix, with signs of bilateral parametrial invasion and superior third of the vagina, in close contact with the bladder and rectum. The biopsy revealed high-grade fusocellular sarcoma. The patient was referred to chemotherapy and neoadjuvant radiotherapy with programming for further surgical treatment. This case corroborates with the thought that although rare, it is important to suggest the possibility of uterine sarcoma in cases of vaginal bleeding after menopause. Histological study and staging are essential to obtain a better therapeutic quality.
\end{abstract}

Key words: uterine hemorrhage, uterine neoplasia, sarcoma.

1 Universidade Estadual do Piauí, Teresina - PI.

2 Faculdade Integral Diferencial, Teresina - PI. * E-mail: raissaprado32@gmail.com 
Resumen: Paciente del sexo femenino, 62 años, con historia de sangrado vaginal en la posmenopausia, abundante e intermitente, con duración de seis meses asociado a la pérdida ponderal importante, dolor pélvico y dificultad miccional. Los exámenes complementarios evidenciaron una lesión expansiva en istmo y cuello uterino, con signos de invasión parametrial bilateral y tercio superior de la vagina, en íntimo contacto con vejiga y recto. La biopsia constató que se trata de sarcoma fusocelular de alto grado. La paciente fue encaminada para quimioterapia y radioterapia neoadyuvante con programación para posterior tratamiento quirúrgico. Este caso corrobora con el pensamiento que, aunque raro, es importante ver la posibilidad de sarcoma uterino, en casos de sangrado vaginal en la posmenopausia. Es fundamental el estudio histológico y la estadificación para obtener una mejor calidad terapéutica.

Palabras clave: hemorragia uterina, neoplasia uterina, sarcoma.

\section{INTRODUÇÃO}

Os sarcomas uterinos constituem um grupo heterogêneo de tumores originados no mesoderma; são raros, correspondendo a cerca de 4 a $9 \%$ das neoplasias malignas uterinas e, aproximadamente, $1 \%$ de todas as malignidades do aparelho genital feminino (Zagouri F, et al., 2009).

Em decorrência da raridade e diversidade patológica dos sarcomas uterinos, ainda existe um conhecimento limitado sobre os mesmos, tornando difícil a individualização de um tratamento otimizado específico (FREITAS F, et al., 2011).

Têm na sua maioria, um mau prognóstico, caracterizando-se por comportamento clínico agressivo, rápida progressão e elevada taxa de metastização à distância; com uma sobrevida global inferior a 50\% em dois anos, mesmo quando diagnosticados precocemente. Devido à baixa incidência e por não apresentarem estágio pré-invasivo, não há uma prática estabelecida para o rastreamento desses tumores. Neste contexto, existe uma quantidade limitada de literatura sobre o mesmo (Schunemann E, et al., 2012).

\section{ESTUDO DE CASO}

Paciente de 62 anos, sexo feminino, casada, do lar, na menopausa há 22 anos. Encaminhada ao serviço de ginecologia de hospital público por dor pélvica que teve início há um ano com piora progressiva. Apresentou ainda, nos últimos seis meses, sangramento vaginal volumoso, sendo necessário o uso contínuo de absorvente noturno, além de indicação médica para receber transfusão de duas bolsas de concentrado de hemácias e subsequente uso de Noripurum ${ }^{\oplus}$ endovenoso em dias alternados. Soma-se ao quadro, perda ponderal estimada de 15 quilos nos últimos cinco meses e a queixa de corrimento transvaginal esverdeado de odor fétido, que passou a se intercalar com o sangramento vaginal. Apresentava ainda, queixa de dificuldade miccional, relacionada a posição adotada (sentada) durante 0 esvaziamento vesical.

Relativo aos antecedentes pessoais, a paciente era hipertensa em uso de captopril $25 \mathrm{mg} / \mathrm{dia}$ e diabética em uso de metformina $1700 \mathrm{mg} /$ dia e glibenclamida $5 \mathrm{mg} /$ dia. Realizou laqueadura tubária há 27 anos. Sobre a história ginecológica, relatava menarca aos 14 anos, três gestações ao longo da vida, sendo todos partos vaginais, nega aborto prévio. Menopausa aos 40 anos. Nega histórico de etilismo e tabagismo.

Ao exame físco: bom estado geral, consciente, orientada, hidratada, hipocorada $(+/+++)$, afebril e normotensa. Ausculta cardiopulmonar fisiológica, abdome globoso, doloroso à palpação profunda, com massa palpável em hipocôndrio, cerca de $4 \mathrm{~cm}$ acima de cicatriz umbilical (correspondente a gestação de aproximadamente 26 semanas).

Exame especular de difícil realização devido a sangramento transvaginal intenso, observou-se lesão friável, de aproximadamente $5 \mathrm{~cm}$, se exteriorizando pelo colo. Ainda durante o exame especular, foi possível a retirada de fragmentos da lesão para biópsia. O resultado do histopatológico em questão foi: sarcoma fusocelular de alto grau. 
Ao toque vaginal: massa de aproximadamente $5 \mathrm{~cm}$, aspecto amolecido, aparente origem uterina, ocupando a topografia do colo, sem comprometimento de paredes vaginais. Paciente com sangramento ativo no momento do exame. Anexos não palpáveis. Toque retal não realizado.

Paciente trazia consigo exames subsidiários. A Ultrassonografia Transvaginal (Figura 1) evidenciou útero em antiversão, centrado de contornos regulares e limites precisos, medindo: $13,4 \times 8,7 \times 13,5 \mathrm{~cm}$ e volume de $818,39 \mathrm{~cm}^{3}$, miométrio de textura acústica homogênea, canal endocervical fechado, anexos não visualizados. Notou-se lesão heterogênea bem definida, volumosa, em região fúndica, não podendo determinar todas as suas medidas pelo método tradicional. A Ultrassonografia de Abdome total, realizada no dia 15/05/2018, revelou lesão heterogênea em cavidade pélvica, de contornos regulares e bem determinados, realizando ejeto de massa, medindo 15,1×9,7×12,6 cm. Foi sugerido na ocasião a realização de Tomografia Computadorizada de abdome (figura 2), para melhor definição da lesão. A mesma foi feita dia 13/06/2018 e mostrou útero globoso de dimensões aumentadas, exibindo material hipodenso heterogêneo no seu interior, cerca de $8,5 \times 7,3 \mathrm{~cm}$, sem repercussões significativas após administração do contraste iodado endovenoso, inespecífico.

No curso de sua internação foi realizada Ressonância Nuclear Magnética de Pelve (25/09/2018), que exibiu útero com dimensões aumentadas, à custa de volumosa lesão expansiva sólida, com realce pelo meio de contraste paramagnético e com restrição à difusão centrada no colo do útero, com sinais de invasão parametrial bilateral e do terço inferior da vagina, sem comprometimento da parede pélvica. A lesão apresentava íntimo contato com a parede posterior da bexiga e parede anterior do reto, com aparente plano de clivagem entre eles e media cerca de 17 × 9,4 x 10,7 cm.

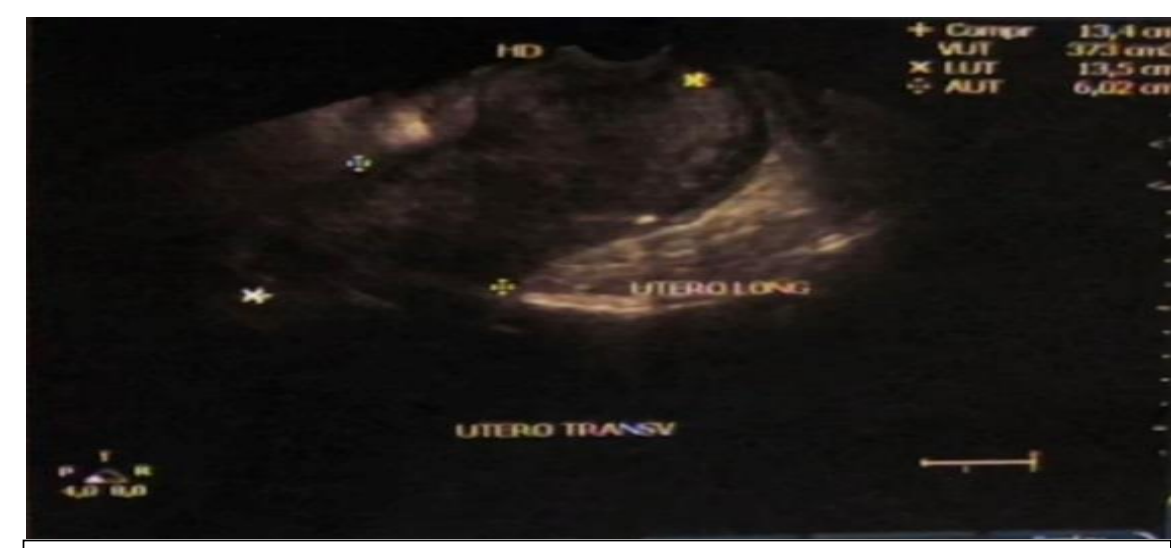

Figura 1: Ultrassonografia Transvaginal. Fonte: Dados da pesquisa.
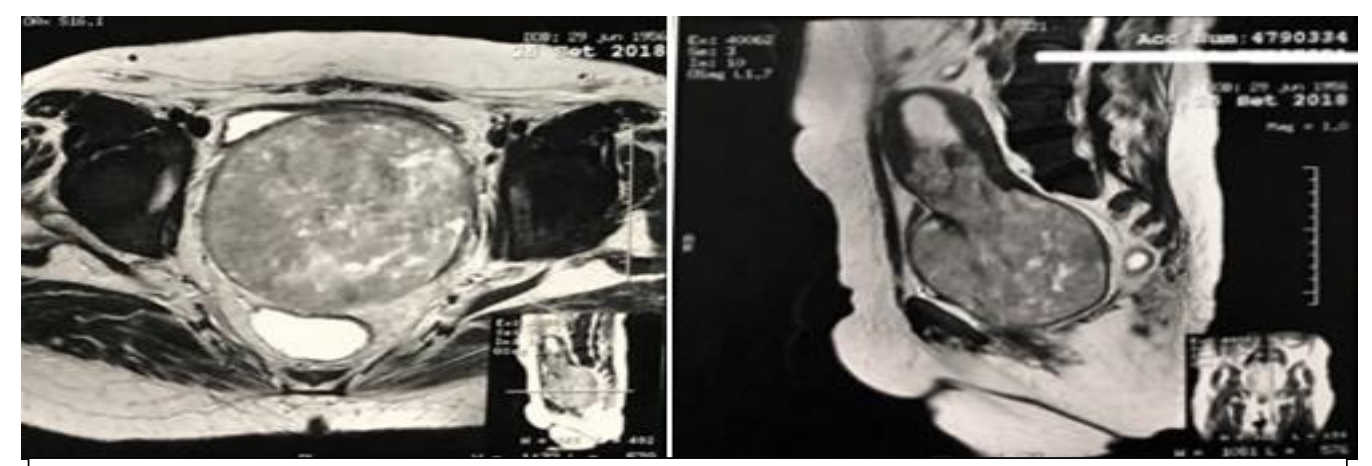

Figura 2 e 3: RNM de pelve. Imagem 2 evidenciando íntima relação com a bexiga. Imagem 3 exibindo extensão tumoral, em istmo e colo uterino, além de ocupar região vaginal. Fonte: Dados da pesquisa. 
Diante do arsenal clínico e complementar, constatou-se extensão tumoral além do corpo uterino e por não haver segurança de um plano de clivagem com a bexiga e reto, optou-se por indicar a terapia neoadjuvante, com programação para posterior tratamento cirúrgico.

\section{DISCUSSÃO}

Inicialmente, os sarcomas uterinos foram histologicamente discriminados em carcinossarcomas, leiomiossarcomas (ambos com incidência aproximada de 40\%), sarcomas estromais endometriais (10\% a $15 \%$ ) e sarcomas indiferenciados (5\% a 10\%) ( SCHUNEMANN E, et al., 2012; D'ANGELO E e PRAT J, 2010).

O carcinossarcoma, também referido como tumor Mulleriano misto maligno (TMMM) é considerado um carcinoma metaplásico, não mais um sarcoma. Assim, com essa nova classificação, o sarcoma uterino mais comum é o leiomiossarcoma (SCHUNEMANN E, et al., 2012; PRAT J, 2009).

Em relação ao grau de diferenciação celular, classificam-se em baixo e alto grau. Os de baixo grau relacionam-se com menor agressividade e recorrência tardia, enquanto que os de alto grau são reconhecidos como sarcomas altamente agressivos, muitas vezes inclassificáveis e nos quais é comum a necrose de coagulação (FREITAS F, et al., 2011; PRAT J, 2009).

Estes provavelmente respondem por cerca de $10 \%$ de todos os sarcomas uterinos. Dada a alta tendência de metástases à distância, paciente pode ser indicada a administração de quimioterapia adjuvante, embora não haja prova de benefício bem estabelecida na literatura (D'ANGELO E, PRAT J, 2010; MADRIGAL M, et al., 2012). No caso relatado neste trabalho, temos um sarcoma fusocelular de alto grau de diferenciação celular (FREITAS F, et al., 2011; REED NS, 2011).

Quanto aos fatores de risco para tumores sarcomatosos do útero, são abordados os que se relacionam a aspectos intrínsecos específicos, tal qual a etnia, apresentando risco aumentado nas mulheres negras; além das mutações genéticas, como é o caso das alterações do gene RB. Outros fatores se relacionam com tratamentos recebidos previamente, incluem-se aqui a radiação pélvica anterior bem como o tratamento a longo prazo com tamoxifeno (KOUHEN F, et al., 2016).

A sintomatologia tem como destaque o sangramento transvaginal (95\% dos casos). Variável em relação a quantidade, pode estar acompanhado de dor, odor fétido ou sintomas de compressão pélvica. As pacientes apresentam graus variados de anemia. Ao exame físico, o útero se encontra aumentado de volume. O crescimento tumoral pode ser tamanho que ocasiona a extrusão de parte da lesão através da cérvice uterina, entidade conhecida como sarcoma parido. Neste caso, todos esses sintomas se fizeram presentes. O principal diagnóstico diferencial de sarcoma é o leiomioma (MADRIGAL M, et al., 2012).

Por possuírem sinais e sintomas semelhantes, a distinção pré-operatória entre as duas entidades em questão pode ser difícil. A malignidade deve ser suspeitada diante da presença de crescimento tumoral nas mulheres na pós-menopausa que não estão usando terapia de reposição hormonal. Tal qual ocorreu na paciente em foco. (PRAT J e MBATANI M, 2015).

Para avaliação inicial em casos suspeitos de sarcoma uterino, tal como ocorreu na investigação em questão, recomenda-se realizar exame físico completo e solicitar biópsia, citologia oncótica, marcador tumoral CA 125 e algum estudo de imagem, seja ressonância magnética e/ou tomografia tóraco-abdominal. Estes últimos com o objetivo de identificar doença extrauterina oculta; uma vez que o principal sítio de metástase é pulmonar. O papel do CA 125 é incerto, sendo mais valorizado para prognóstico do que para o diagnóstico em si. O diagnóstico definitivo é apenas intraoperatório. O caso em estudo possuiu investigação semelhante, não sendo realizada apenas CA 125 e citologia (tecnicamente impossível de ser realizada devido a presença do tumor na região) (MADRIGAL M, et al., 2012). 
O estadiamento de um sarcoma uterino é fator primordial na seleção do tratamento e leva em consideração a extensão tumoral no útero, bem como sua metastização. O caso apresentado neste artigo foi classificado no estádio IIB baseado nos dados de estadiamento da FIGO para sarcomas uterinos, pois ocorre o envolvimento de outros tecidos pélvicos, avaliado em consonância com as imagens representadas em RNM da pelve (PRAT J e MBATANI M, 2015).

A base do tratamento consiste na terapia cirúrgica. Porém, devido a combinação de baixa incidência e diversidade histopatológica dos sarcomas, existe pouco consenso em uma terapia efetiva para a totalidade das apresentações existentes. A histerectomia acompanhada de salpingectomia e ooforectomia seriam os pontos de partida para o tratamento da lesão. A ooforectomia deve ser ponderada de acordo com a idade da paciente e o estádio da doença. A linfadenectomia não é consenso, efetivamente recomendada em casos de aumento ganglionar. Recomenda-se ainda, realização de lavado peritoneal e citologia em órgãos abdominais e cúpula diafragmática. Deve-se avaliar individualmente os benefícios de terapia neoadjuvante com quimioterapia ou radioterapia, partindo de preceitos como: a ressecabilidade da lesão e a disseminação a distância da doença. No caso em relato, devido a grande extensão da lesão, o duvidoso plano de clivagem com a parede posterior da bexiga e anterior do reto, optou-se pelo encaminhamento à quimio e radioterapia neoadjuvante e posterior citorredução máxima ciríurgica (REED NS, 2008).

No que se refere ao seguimento subsequente ao tratamento cirúrgico, recomenda-se a realização de tomografia computadorizada de Tórax / abdome / pelve a cada 3-6 meses nos primeiros 3 anos e, depois, a cada 6-12 meses nos próximos 2 anos. Dependendo do grau histológico no estadiamento inicial, considerar a geração de imagens anual ou bi-anualmente a partir dos 5 anos. A ressonância nuclear magnética, com a mesma periodicidade da tomografia computadorizada, é opcional. A PET/TC deve ser considerada nos casos suspeitos de metástases.

\section{CONSIDERAÇÕES FINAIS}

Apesar da baixa incidência, os sarcomas uterinos, devem ser aventados como possibilidade diagnóstica, principalmente em situações de sangramento transvaginal na pós menopausa, acompanhado de aumento abdominal. Dada a agressividade da patologia em questão, sintomas sistêmicos também floreiam o quadro clínico. Exames de imagem desempenham papel fundamental no estadiamento, bem como a realização de biópsia e o estudo histopatológico da amostra. Este relato de caso, vem evidenciar a importância desde a suspeição clínica, até a solicitação de corretos exames de imagem, como o pronto diagnóstico e estadiamento da lesão. Embora, não existindo consenso na literatura estudada acerca de um padrão único de tratamento, buscou-se evidências literárias e uma apurada interpretação de exames complementares para subsidiar a decisão de implantar terapia neoadjuvante precoce ao tratamento definitivo, que seria a citorredução cirúrgica.

\section{REFERÊNCIAS}

1. AMERICAN CANCER SOCIETY. UTERINE SARCOMA. Atlanta, Ga: American Cancer Society; 2013.

2. D'ANGELO E, PRAT J. Uterine sarcomas: a review. Gynecol Oncol. 2010; 116(1): 131-139.

3. FREITAS F, et al. Rotinas em Ginecologia, $20116^{\mathrm{a}}$ ed. Porto Alegre: Artmed

4. KOUHEN F, et al. Tamoxifen Treatment and Risk of Endometrial Cancer: An Overview. Journal of Health Science 4. 2016. 1-7 doi: 10.17265/2328-7136/2016.01.001

5. MADRIGAL M, et al. Sarcoma Uterino: Revisión de la literatura. GAMO. marzo - abril 2012; 11/2.

6. National Comprehensive Cancer Network. NCCN Clinical Practice Guidelines in Oncology: Uterine Neoplasms. NCCN Guidelines. 2018, may 25. 2.2018

7. PRAT J. FIGO staging for uterine sarcomas. International Journal of Gynecology and Obstetrics. 2009.

8. PRAT J, MBATANI N. Uterine sarcomas. FIGO câncer Report 2015. International Journal of Gynecology and Obstetrics. 0020-7292.

9. REED NS, et al. Phase III randomised study to evaluate the role of adjuvant pelvic radiotherapy in the treatment of uterine sarcomas stages I and II: An European Organisation for Research and Treatment of Cancer Gynaecological Cancer Group Study. Eur J Cancer 2008; 44(6): 808-818.

10. REED NS. The management of uterine sarcomas. Clin Oncol (R Coll Radiol). 2008; 20(6):470-478.

11. SCHUNEMANN E, et al. Novos conceitos e revisão atualizada sobre sarcomas uterinos - New concepts and update review of uterine sarcomas. Femina; 2012; 40(3).

12. ZAGOURI F, et al. Treatment of early uterine sarcomas: disentangling adjuvant modalities. World Journal of Surgical Oncology. 2009 7:38. doi:10.1186/1477-7819-7-38 